\title{
The Different Ways of Stabbing Disjoint Convex Sets*
}

\author{
M. Katchalski, ${ }^{1}$ T. Lewis, ${ }^{2}$ and A. Liu ${ }^{2}$ \\ ${ }^{1}$ Department of Mathematics, Technion, Haifa, Israel \\ ${ }^{2}$ Department of Mathematics, University of Alberta, Edmonton, Canada
}

\begin{abstract}
We construct a family of $n$ disjoint convex set in $\mathbb{R}^{d}$ having $(n /(d-1))^{d-1}$ geometric permutations. As well, we complete the enumeration problem for geometric permutations of families of disjoint translates of a convex set in the plane, settle the case for cubes in $\mathbb{R}^{d}$, and construct a family of $d+1$ translates in $\mathbb{R}^{d}$ admitting $(d+1) ! / 2$ geometric permutations.
\end{abstract}

\section{Introduction}

A stabbing line, or common transversal, for a family $\mathscr{A}$ of sets in $\mathbb{R}^{d}$ is a straight line that intersects every member of $\mathscr{A}$. If $\mathscr{A}$ is finite and if the members of $\mathscr{A}$ are convex and pairwise disjoint, then a common transversal meets the members of $\mathscr{A}$ in a definite order. The transversal thus determines two permutations of $\mathscr{A}$, one being the reverse of the other. The pair of permutations is called a geometric permutation or a G.P. of $\mathscr{A}$. Figure 1 gives examples of families admitting exactly one, two, three, and six different geometric permutations.

Geometric permutations were introduced in [6] and [8] where an example was given of $n$ convex sets in the plane having $2 n-2$ different G.P.s, and where it was shown that for $n$ pairwise disjoint convex sets the number of G.P.s cannot exceed $\left(\begin{array}{l}n \\ 2\end{array}\right)$. Wenger [10] tightened the upper bound by showing that the number of G.P.s cannot exceed $6 n+6$, and Edelsbrunner and Sharir [2] have completely closed the gap by showing that the maximum number is in fact $2 n-2$.

The situation in higher dimensions is of some interest. In [8] we claimed that the number of G.P.s for $n$ disjoint convex sets in $\mathbb{R}^{d}$ may exceed $n^{d-1}$ times a

\footnotetext{
* This research was partly supported by NSERC Grants A3062, A5137, and A8761.
} 

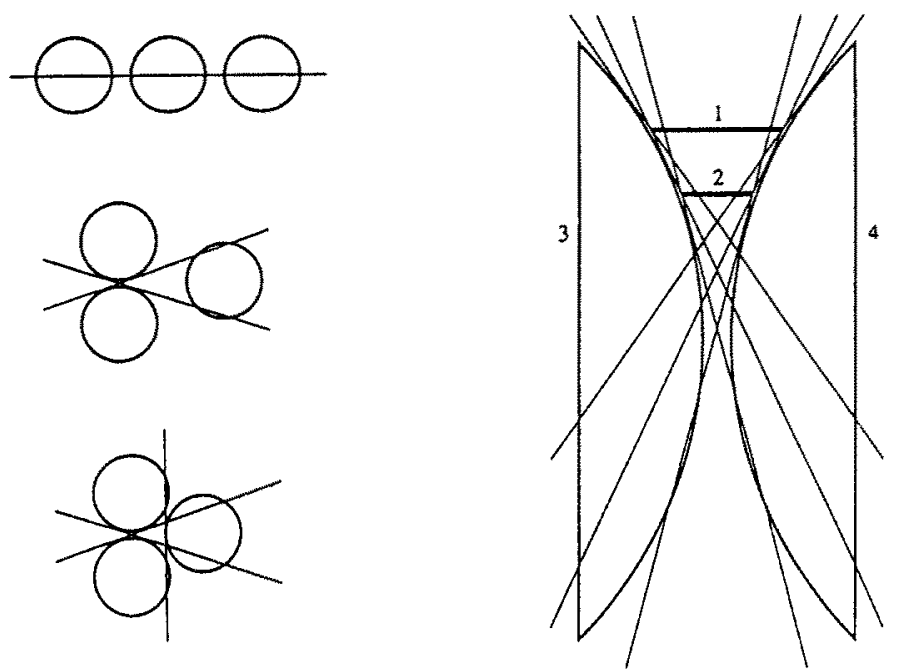

Fig. 1. Families with exactly one, two, three, and six geometric permutations.

constant. As pointed out in [2] and [10], as yet this is still just a claim, and so in this note we provide a proof.

In the plane, a family consisting of mutually disjoint translates of a compact convex set cannot have such a large number of G.P.s. In [7] we showed that, with some possible exceptions, the number of G.P.s cannot exceed three. Here, we show that the result is true without any exceptions.

We also examine families of translates in higher dimensions. We show that, in $\mathbb{R}^{d}$, a family of disjoint translates of a cube can have no more than $2^{d-1}$ G.P.s. An example is given to show that a family of cubes can actually have this many G.P.s. For arbitrary families of translates, this number is far from an upper bound-we provide an example showing that a family of disjoint translates of a compact convex set may admit $(d+1) ! / 2$ G.P.s.

The study of geometric permutations has been motivated by a theorem of Hadwiger [4] which states that if a disjoint family of compact convex sets in the plane can be ordered in such a way that every three members of the family admit a transversal in the specified order, then there is a transversal for the entire family.

Hadwiger's theorem relates orderings of the sets to common transversals, and it was hoped that more information on G.P.s would yield results on the existence of stabbing lines. This has proved to be the case: see the results in [5] and those of Tverberg [9]. These two papers have used geometric permutations to provide an affirmative answer to a conjecture of Grünbaum [3] that a family of disjoint translates of a convex set in the plane has a common transversal if every five members of the family have a common transversal.

\section{Geometric Permutations in $d$ Dimensions}

In this section we show that it is possible for $n$ pairwise disjoint compact convex sets in $\mathbb{R}^{d}$ to have $(n /(d-1))^{d-1}$ geometric permutations. 




Fig. 2. A family of six sets in $\mathbb{R}^{3}$ admitting nine geometric permutations.

Since we are dealing with a finite family of sets and a finite number of common transversals, we note that there is no loss in generality if the sets are unbounded. (If some sets are unbounded, then choose one point from the intersection of each set with each stabbing line. The collection of all of these points is bounded, and so we can create a bounded family with the same number of geometric permutations by intersecting the family with a ball of sufficiently large radius.)

We mention that there is also no real loss if some of the sets are not closed, provided each transversal intersects the relative interior of each nonclosed set. (A proof follows from the fact that if $p$ is a relative interior point of a convex set $C$, then, for any $\mu$ with $0<\mu<1$, the closure of $p+\mu(C-p)$ is a subset of $C$.)

The family of $n$ sets is comprised of $d-1$ closed cylinders together with a collection of $(m-1)(d-1)$ relatively open parallel plates. Figure 2 depicts the situation for $d=3$ and $m=3$ (the common transversals are not shown). The cylinders are given by

$$
C_{i}=\left\{x: x_{i}^{2}+\left(x_{d}-3 i\right)^{2} \leq 1\right\}, \quad i=1,2, \ldots, d-1 .
$$

The plates are half-hyperplanes of the form

$$
\begin{aligned}
& P_{i, j}=\left\{x: x_{d}=3 i+j / 2 m, x_{i}>\sqrt{1-\left(x_{d}-3 i\right)^{2}}\right. \\
& i=1,2, \ldots, d-1 ; j=1,2, \ldots, m-1 .
\end{aligned}
$$

No two cylinders and no two plates intersect since their projections onto the $x_{d}$ axis do not intersect. For the same reason, when $i \neq i^{\prime}$, the cylinder $C_{i}$ and the plate $P_{i, j}$ do not intersect. Finally, $C_{i}$ and $P_{i, j}$ do not intersect because $x_{i} \leq$ $\sqrt{1-\left(x_{d}-3 i\right)^{2}}$ in $C_{i}$ while $x_{i}>\sqrt{1-\left(x_{d}-3 i\right)^{2}}$ in $P_{i, j}$.

For $i=1,2, \ldots, d-1$ and $j=1,2, \ldots, m$, let $H_{i, j}$ be the hyperplane tangent to the cylinder $C_{i}$ at the $(d-2)$-flat

$$
\left\{x: x_{d}=3 i+j / 2 m-1 / 4 m, x_{i}=\sqrt{1-\left(x_{d}-3 i\right)^{2}}\right\} .
$$


The intersection of $H_{1, j_{1}}, H_{2, j_{2}}, \ldots, H_{d-1, j_{d-1}}$ is a line passing through all cylinders and plates. Each choice of $j_{k}$ 's gives rise to a different geometric permutation. There are $m^{d-1}$ such choices, and so the cylinders and plates admit $(n /(d-1))^{d-1}$ geometric permutations.

\section{Geometric Permutations of Translates}

In [7] it was shown that, in the plane, a family of pairwise disjoint translates of a compact convex set has at most three G.P.s, except possibly for families of size five or six. In this section we show that the result remains true even for families containing five or six sets.

If a line meets the sets $S_{i_{1}}, S_{i_{2}}, \ldots, S_{i_{k}}$ in that order, then the resulting geometric permutation may be denoted using either $\left\langle i_{1}, i_{2}, \ldots, i_{k}\right\rangle$ or $\left\langle i_{k}, i_{k-1}, \ldots, i_{1}\right\rangle$. In [7] it was shown that certain pairs of geometric permutations cannot coexist. We state these facts here as lemmas, but omit the proofs. (In all of the lemmas, by a family of translates it is understood to mean a family of pairwise disjoint translates of a given compact convex set.)

Lemma 3.1. If $\langle i, j, k, l\rangle$ is a G.P. for a family of translates, then $\langle j, i, l, k\rangle$ is not.

Lemma 3.2. If $\langle i, j, k, l\rangle$ is a G.P. for a family of translates, then $\langle i, l, k, j\rangle$ is not.

It should be mentioned that although Lemma 3.1 is stated in the context of a family of translates, it remains true for any family of pairwise disjoint compact convex sets. However, Lemma 3.2, as well as the ones below, fails for families that are not translates.

We compare different G.P.s by using their representations. We say that the representations $\left\langle u_{1}, \ldots, u_{n}\right\rangle$ and $\left\langle v_{1}, \ldots, v_{n}\right\rangle$ are $K$-consistent if $u_{i}=v_{i}$ except possibly for $i \in\{j, j+1, \ldots, j+k-1\}$ where $k$ is some positive integer with $k \leq n$. In other words, the two representations are $k$-consistent if they are identical except in at most $k$ consecutive places.

Since each G.P. has two possible representations, it is clear that the notion of $k$-consistency depends upon which representatives are chosen. The following two results were also obtained in [7]:

Lemma 3.3. Given any two G.P.s for a family of translates, it is possible to choose representatives that are 4-consistent.

Lemma 3.4. If all G.P.s for a family of translates can be represented by permutations that are pairwise 4-consistent, then the family has at most three G.P.s.

In order to show that a family of translates of five or six sets has at most three G.P.s, we will show that such families satisfy the hypothesis of Lemma 3.4. The proofs for the two cases are somewhat different and are presented separately. 
Theorem 3.5. All G.P.s for a family of five translates may be represented by permutations that are pairwise 4-consistent.

Proof. Let $p=\langle 1,2,3,4,5\rangle$ be a representation for a G.P. for the family of translates. By Lemma 3.3, for every other G.P. it is possible to choose a representative that is 4-consistent with $p$. We will show that any two such representatives, say $u=\left\langle u_{1}, \ldots, u_{5}\right\rangle$ and $v=\left\langle v_{1}, \ldots, v_{5}\right\rangle$, are 4-consistent with each other.

Now, by 4-consistency with $p$, either $u_{1}=1$ or $u_{5}=5$ and likewise either $v_{1}=1$ or $v_{5}=5$. If we suppose that $u$ is not 4-consistent with $v$, then $u$ is 4-consistent with $-v=\left\langle v_{5}, \ldots, v_{1}\right\rangle$. In this case it is impossible that either $u_{1}=1=v_{1}$ or $u_{5}=5=v_{5}$. By symmetry, we may assume that $u_{1}=1 \neq v_{1}$ and $u_{5} \neq 5=v_{5}$. Then we must have $u_{5}=v_{1} \in\{2,3,4\}$. We show that each of these three possibilities cannot occur.

Case I: $u_{5}=v_{1}=2$. Let $i, j \in\{2,3,4\}$, with $i<j$. If $u_{i}>u_{j}$, then from $u$ we obtain the G.P. $\left\langle 1, u_{i}, u_{j}, 2\right\rangle$, while from $p$ we get the G.P. $\left\langle 1,2, u_{j}, u_{i}\right\rangle$, which contradicts Lemma 3.2. This leaves only the possiblity that $u=\langle 1,3,4,5,2\rangle$. Then from $u$ we obtain the G.P. $\langle 3,4,5,2\rangle$ while from $p$ we obtain $\langle 2,3,4,5\rangle$, which contradicts Lemma 3.2 .

Case II: $u_{5}=v_{1}=3$. Let $u_{i}=4$ and $u_{j}=5$. If $i>j$, then $u$ yields the G.P. $\langle 1,5,4,3\rangle$ while $p$ contains $\langle 1,3,4,5\rangle$, contradicting Lemma 3.2. So $u$ is one of $\langle 1,2,4,5,3\rangle,\langle 1,4,2,5,3\rangle$, or $\langle 1,4,5,2,3\rangle$. However, the last one yields the G.P. $\langle 4,5,2,3\rangle$, and $p$ yields $\langle 2,3,4,5\rangle$, which is impossible by Lemma 3.1. It follows that $u$ must contain the G.P. $\langle 1,2,5,3\rangle$.

Now let $v_{i}=1$ and $v_{j}=2$. If $\left.i\right\rangle j$, then from $v$ we obtain the G.P. $\langle 3,2,1,5\rangle$, and from $p$ we obtain $\langle 1,2,3,5\rangle$, which is impossible by Lemma 3.2. So we must have $i<j$, showing that $v$ yields $\langle 3,1,2,5\rangle$, but this, together with the G.P. $\langle 1,2,5,3\rangle$ from $u$, contradicts Lemma 3.2 .

Case III: $u_{5}=v_{1}=4$. The argument for this case parallels that for Case I.

Theorem 3.6. All G.P.s for a family of six translates may be represented by permutations that are pairwise 4-consistent.

Proof. We may assume that $p=\langle 1,2,3,4,5,6\rangle$ is a representation for one of the G.P.s for the family of translates. As in the previous proof, by Lemma 3.3 it is possible to choose representatives for all other G.P.s that are 4-consistent with $p$. We will show that any two such representatives, say $u=\left\langle u_{1}, \ldots, u_{6}\right\rangle$ and $v=\left\langle v_{1}, \ldots, v_{6}\right\rangle$, are 4-consistent with each other.

Again, by 4 -consistency with $p$, either $u_{1}=1$ or $u_{6}=6$ and likewise either $v_{1}=1$ or $v_{6}=6$. Suppose that $u$ is not 4-consistent with $v$. Then $u$ is 4-consistent with $-v=\left\langle v_{6}, \ldots, v_{1}\right\rangle$. As a consequence, it is impossible that either $u_{1}=1=v_{1}$ or $u_{6}=6=v_{6}$. By symmetry, we may assume that $u_{1}=1 \neq v_{6}$ and $u_{6} \neq 6=v_{6}$. Since $u_{6} \neq 6$ in order that $u$ and $p$ be 4-consistent we must have $u_{2}=2$. Similarly, we must have $v_{5}=5$. Since $u$ and $-v$ are 4-consistent, and since $u_{1} \neq v_{6}$, we must have $u_{5}=v_{2}$ and $u_{6}=v_{1}$, and these values must be 3 and 4 . 
If $u_{5}=v_{2}=3$, then $u_{6}=v_{1}=4$, and we obtain G.P.s $\langle 6,2,3,4\rangle$ from $v$ and $\langle 2,3,4,6\rangle$ from $p$. If $u_{5}=v_{2}=4$, then $u_{6}=v_{1}=3$, and obtain G.P.s $\langle 1,5,4,3\rangle$ from $u$ and $\langle 1,3,4,5\rangle$ from $p$. Either case contradicts Lemma 3.2.

\section{Examples for Translates in $d$ Dimensions}

One of the earliest results about common transversals was obtained by Santalo [1], [4], and dealt with families of parallelotopes whose edges were parallel to the coordinate axes (an isothetic family).

Theorem 4.1. An isothetic family of pairwise disjoint parallelotopes in $\mathbb{R}^{\mathrm{d}}$ admits at most $2^{d-1}$ G.P.s.

Proof. The proof uses an idea employed by Wenger in [10]. Any two of the parallelotopes can be separated by a translate of one of the coordinate hyperplanes. Consequently, two transversals of the form $x=p+\lambda a$ and $y=q+\lambda b$ will result in the same G.P. if $a$ and $b$ belong to the same $d$-dimensional orthant. The theorem now follows because every line in $\mathbb{R}^{d}$ is parallel to a vector in one of $2^{d-1}$ orthants.

The following shows that the upper bound is tight.

Theorem 4.2. In $\mathbb{R}^{d}, d=2,3, \ldots$, there is a family of $d+1$ disjoint translates of the unit cube that admits $2^{d-1}$ G.P.s.

Proof. We construct the family inductively, beginning with $d=2$. The family in $\mathbb{R}^{2}$ is shown in Fig. 3(a). In fact, we use a process similar to that in Section 2. Rather than actually fit the cubes in place, we construct a family of unbounded

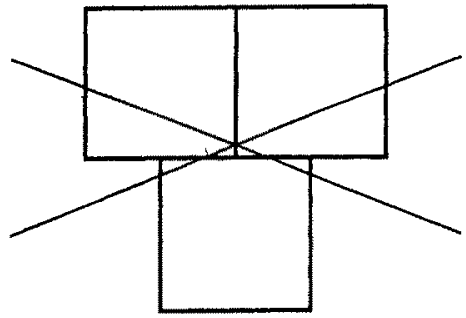

(a)

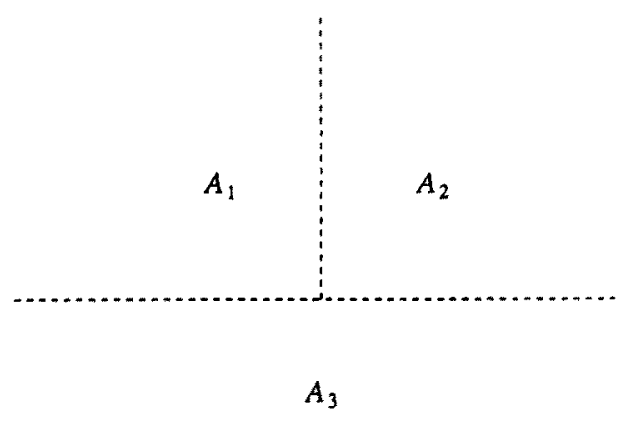

(b)

Fig. 3. (a) Translates of three "cubes" in $\mathbb{R}^{2}$ admitting two G.P.s. (b) Associated unbounded sets $A_{1}$, $A_{2}, A_{3}$ (see the proof of Theorem 4.2). 
open sets each of which is of the type

$$
\left\{\left(x_{1}, x_{2}, \ldots, x_{d}\right): x_{j} \gtrless 0, j=i_{1}, \ldots, i_{m}\right\},
$$

where $\gtrless$ denotes either "<" or " $>$." In $\mathbb{R}^{2}$ the sets (see Fig. 3(b)) are

$$
\begin{aligned}
& A_{1}=\left\{\left(x_{1}, x_{2}\right): x_{1}<0, x_{2}>0\right\}, \\
& A_{2}=\left\{\left(x_{1}, x_{2}\right): x_{1}>0, x_{2}>0\right\}, \\
& A_{3}=\left\{\left(x_{1}, x_{2}\right): x_{2}<0\right\} .
\end{aligned}
$$

If a straight line in $\mathbb{R}^{d}$ intersects the $d+1$ sets $A_{i}$, it is evident that we can find a cube of a sufficiently large size, say $Q=\left\{\left(x_{1}, \ldots, x_{d}\right):\left|x_{j}\right|<M, 1 \leq j \leq d\right\}$, such that the line intersects all of the sets $A_{i} \cap Q$. Now, for each set $A_{i} \cap Q$, there is a translate $v_{i}+Q$ of $Q$ such that

$$
A_{i} \cap Q \subset v_{i}+Q \subset A_{i}
$$

and this shows that there is no loss in generality in dealing with open bounded sets of the type defined by (1). The proof that these sets admit $2^{d+1}$ geometric permutations is straightforward and is left to the reader.

If we do not distinguish between a permutation and its reversal, the maximum number of permutations of $n$ objects is $n ! / 2$. Whether this can be achieved for geometric permutations of $n$ mutually disjoint translates of a convex set depends upon the shape of the set. For example, Theorem 4.1 shows that it is not possible with cubes in $\mathbb{R}^{d}$, while Fig. 1 demonstrates that in $\mathbb{R}^{2}$ it is possible with $n=3$ circles. We will show that a similar result holds in $\mathbb{R}^{d}$, that is, there is a family of $n=d+1$ translates admitting $n ! / 2$ geometric permutations. We begin with the following lemma:

Lemma 4.3. Let $V=\left\{v_{1}, v_{2}, \ldots, v_{d+1}\right\}$ be the vertices of a simplex whose centroid is the origin, and let $K_{i}$ be the convex cone spanned by $V \backslash\left\{v_{i}\right\}$. There is a straight line that intersects the interior of each of $K_{1}, \ldots, K_{d+1}$.

Proof. Let $\alpha>0$ be fixed. Let $L$ be the straight line whose equation is $x=p+\lambda q$, $-\infty<\lambda<\infty$, where

$$
p=\sum_{j=1}^{d+1} \alpha^{2 j} v_{j} \text { and } q=-\sum_{j=1}^{d+1} \alpha^{j} v_{j}
$$

This line intersects the interior of each $K_{i}$. To check this, let $\lambda_{i}=2 \alpha^{i}$, and write $p+\lambda_{i} q$ as $\sum_{j=1}^{d+1} \gamma_{j} v_{j}$. The coefficients are $\gamma_{j}=\alpha^{2 j}-2 \alpha^{i} \alpha^{j}$, and when $j=i$ we have $\gamma_{i}=-\alpha^{2 i}$. Then, for $j \neq i$,

$$
\gamma_{j}-\gamma_{i}=\alpha^{2 j}-2 \alpha^{i} \alpha^{j}+\alpha^{2 i}=\left(\alpha^{j}-\alpha^{i}\right)^{2}>0
$$


showing that $\gamma_{j}$ is strictly larger than $\gamma_{i}$. Then, since $\sum_{j=1}^{d+1} v_{j}=\overline{0}$,

$$
p+\lambda_{i} q=\sum_{j=1}^{d+1} \gamma_{j} v_{j}-\gamma_{i} \sum_{j=1}^{d+1} v_{j}=\sum_{j \neq i, j=1}^{d+1}\left(\gamma_{j}-\gamma_{i}\right) v_{j}
$$

which shows that $p+\lambda_{i} q$ is interior to $K_{i}$.

Theorem 4.4. In $\mathbb{R}^{d}$ there is a family of disjoint translates of size $d+1$ that admits $(d+1) ! / 2$ geometric permutations.

Proof. Let $v_{0}=\overline{0}$ and let $v_{1}, v_{2}, \ldots, v_{d+1}$ be the vertices of an equilateral simplex whose centroid is the origin in $\mathbb{R}^{d}$. Let $A$ be the interior of the convex hull of $\left\{v_{i}+v_{j}: 0 \leq i, j \leq d+1 ; i \neq j\right\}$. We will show that the family $\left\{A-v_{i}: 1 \leq i \leq\right.$ $d+1\}$ is pairwise disjoint and admits $(d+1) ! / 2$ geometric permutations. (In $\mathbb{R}^{2}$, $A$ is a hexagon; in $\mathbb{R}^{3}$ it is the polyhedron shown in Fig. 4(c). As well as $A$, we may use the interior of the convex hull of

$$
\left\{k v_{i}+v_{j}: 1 \leq i \leq d+1 ; 0 \leq j \leq d+1 ; j \neq i\right\}
$$

where $k \geq 1$ is constant. A polyhedron of this type is used in Fig. 4(a) and (b).)

To verify that $A-v_{m}$ and $A-v_{n}$ are disjoint, note that $A-v_{n}$ is the interior of the convex hull of $\left\{v_{i}+v_{j}-v_{n}: 0 \leq i, j \leq d+1 ; i \neq j\right\}$. Letting $(\cdot, \cdot)$ denote the inner product, and using the fact that $\left(v_{i}, v_{j}\right)=c<0$ when $v_{i}$ and $v_{j}$ are different

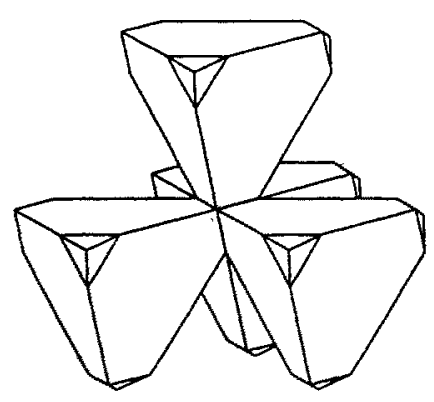

(a)

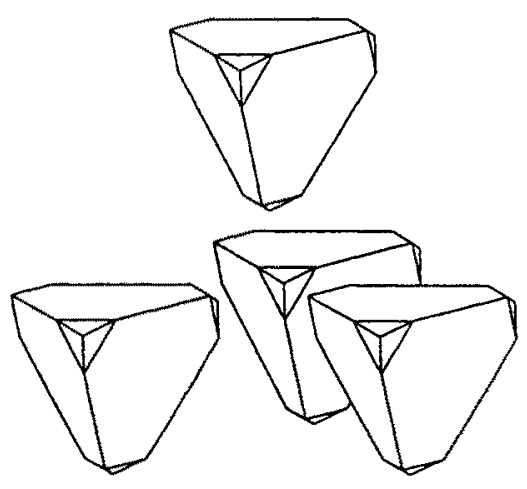

(b)



(c)

Fig. 4. (a) A family of four translates in $\mathbb{R}^{3}$ admitting 12 geometric permutations (b) An "exploded view" of the family. (c) The set used in the proof of Theorem 4.4. 
vertices of the equilateral simplex, we have

$$
\left(v_{m}-v_{n}, v_{i}+v_{j}-v_{n}\right)= \begin{cases}\left\|v_{m}\right\|^{2}-\left(v_{m}, v_{n}\right), & \{i, j\}=\{m, n\}, \\ \left\|v_{m}-v_{n}\right\|^{2}, & \{i, j\} \cap\{m, n\}=\{m\}, \\ 0, & \{i, j\} \cap\{m, n\}=\{n\}, \\ \left\|v_{n}\right\|^{2}-\left(v_{m}, v_{n}\right), & \text { otherwise. }\end{cases}
$$

This shows that $\inf \left(v_{m}-v_{n}, A-v_{n}\right)=0$, and, by interchanging $m$ and $n$, that $\inf \left(v_{n}-v_{m}, A-v_{m}\right)=0$, that is, $\sup \left(v_{m}-v_{n}, A-v_{m}\right)=0$, showing that $A-v_{n}$ and $A-v_{m}$ are disjoint.

We also note that the convex cone, $K^{\prime}$, generated by $A-v_{n}$ is the same as the cone $K_{n}$ defined in Lemma 4.3. To verify this, note that $K^{\prime}$ is generated by $\left\{v_{i}+v_{j}-v_{n}: 0 \leq i, j \leq d+1 ; i \neq j\right\}$. Letting $v_{j}=v_{n}$, we see that $v_{i} \in K^{\prime}$, showing that $K_{n} \subset K^{\prime}$. On the other hand, if either $v_{i}$ or $v_{j}$ equals $v_{n}$, then we have $v_{i}+v_{j}-v_{n} \in K_{n}$. If neither $v_{i}$ nor $v_{j}$ equals $v_{n}$ then, noting that $-v_{n}=\sum_{j \neq n} v_{j}$, it is evident that $v_{i}+v_{j}-v_{n}$ is a nonnegative linear combination of $v_{i}, i=1, \ldots$, $n-1, n+1, \ldots, d+1$, and again we have $v_{i}+v_{j}-v_{n} \in K_{n}$. Thus, $K^{\prime} \subset K_{n}$.

Because of symmetry, to show that the family $\left\{A+v_{i}: 1 \leq i \leq d+1\right\}$ has $(d+1) ! / 2$ geometric permutations, it suffices to show that it has one geometric permutation. This follows from Lemma 4.3, and completes the proof of the theorem.

Questions. 1. Is it true that the maximum number of G.P.s for any family of disjoint translates in $\mathbb{R}^{d}$ is $(d+1) ! / 2$ ? Although we conjecture that this is so, except for the case where $d=2$, it is not even known if there is an upper bound that is independent of the size of the family.

2. A similar question arises for arbitrary disjoint families in $\mathbb{R}^{d}$, that is, is the lower bound of $C \cdot n^{d-1}$ given in Section 2 also an upper bound for a family of $n$ compact convex sets? The constant $C$ in the example is $(d-1)^{-(d-1)}$. Is the result true with a constant that does not depend upon the dimension?

Very relevant to both of the questions is a result of Wenger who showed in [10] that a family of $n$ pairwise disjoint compact convex sets admits at most $O\left(n^{2 d-2}\right)$ geometric permutations.

\section{Acknowledgments}

The authors would like to thank the referees for their very useful comments and suggestions.

\section{References}

1. L. Danzer, B. Grünbaum, and V. Klee, Helley's Theorem and Its Relatives, Proceedings of Symposia in Pure Mathematics, Vol. 7, American Mathematical Society, Providence, RI, 1962, pp. 101-180. 
2. H. Edelsbrunner and $M$. Sharir, The maximum number of ways to stab $n$ convex nonintersecting objects in the plane is $2 n-2$, Discrete Comput. Geom. 5 (1990), $35-42$.

3. B. Grünbaum, On common transversals, Arch. Math. 9 (1958), $465-469$.

4. H. Hadwiger, H. Debrunner, and V. Klee, Combinatorial Geometry in the Plane, Holt, Rinehart and Winston, New York, 1964.

5. M. Katchalski, A conjecture of Grünbaum on common transversals, Math. Scand. 59 (1986), 192-198.

6. M. Katchalski, T. Lewis, and A. Liu, Geometric permutations and common transversals, Discrete Comput. Geom. 1 (1986), 371-377.

7. M. Katchalski, T. Lewis, and A. Liu, Geometric permutations of disjoint translates of convex sets, Discrete Math 65 (1987), 249-259.

8. M. Katchalski, T. Lewis, and J. Zaks, Geometric permutations for convex sets, Discrete Math. 54 (1985), 271-284.

9. H. Tverberg, Proof of Grünbaum's conjecture on common transversals for translates, Discrete Comput. Geom. 4 (1989), 191-203.

10. R. Wenger, Upper bounds on geometric permutations, Discrete Comput. Geom. 5 (1990), 27-33.

Received January 22, 1990, and in revised form July 25, 1991. 